\title{
Tobacco Chewing and Adult Mortality: a Case-control Analysis of 22,000 Cases and 429,000 Controls, Never Smoking Tobacco and Never Drinking Alcohol, in South India
}

\author{
Vendhan Gajalakshmi*, Vendhan Kanimozhi
}

\begin{abstract}
Background: Tobacco is consumed in both smoking and smokeless forms in India. About 35-40\% of tobacco consumption in India is in the latter. The study objective was to describe the association between chewing tobacco and adult mortality. Materials and Methods: A case-control study was conducted in urban (Chennai city) and rural (Villupuram district) areas in Tamil Nadu state in South India. Interviewed in 1998-2000 about 80,000 families $(48,000$ urban and 32,000 rural) with members who had died during 1995-1998. These were the cases and their probable underlying cause of death was arrived at by verbal autopsy. Controls were $600,000(500,000$ urban, 100,000 rural) individuals from a survey conducted during 1998-2001 in the same two study areas from where cases were included. Results: Mortality analyses were restricted to non-smoking non-drinkers aged 35-69. The age, sex, education and study area adjusted mortality odds ratio was 30\% higher (RR:1.3, 95\% CI:1.2-1.4) in ever tobacco chewers compared to never chewers and was significant for deaths from respiratory diseases combined (RR:1.5,95\% CI:1.4-1.7), respiratory tuberculosis (RR:1.7,95\% CI:1.5-1.9), cancers all sites combined (RR:1.5, 95\% CI:1.4-1.7) and stroke (RR:1.4, 95\% CI:1.2-1.6). Of the cancers, the adjusted mortality odds ratio was significant for upper aero-digestive, stomach and cervical cancers. Chewing tobacco caused $7.1 \%$ of deaths from all medical causes. Conclusions: The present study is the first large study in India analysing non-smoking non-drinkers. Statistically significant excess risks were found among ever tobacco chewers for respiratory diseases combined, respiratory tuberculosis, stroke and cancer (all sites combined) compared to never tobacco chewers.
\end{abstract}

Keywords: Tobacco chewing - smokeless tobacco - mortality - tuberculosis - cervical cancer

Asian Pac J Cancer Prev, 16 (3), 1201-1206

\section{Introduction}

Tobacco consumption in any form, smoking or smokeless, is a major source of premature mortality. About $20 \%$ of global tobacco related mortality occurs in India (World Health Organization, 1999). Globally, among adults, $27 \%$ of males and $9 \%$ of females use smokeless tobacco tobacco (Global progress report 2010). Among those aged 15 or older, $28 \%$ of males and $12 \%$ of females were earlier found to use smokeless tobacco. The higher prevalence of tobacco chewing is noted less educated group, in rural population than in urban and in women compared to men (Gajalakshmi et al., 2012; Thakpur et al., 2013).

For this study, we have used data from two large household surveys conducted in South India. One is a retrospective enquiry of the habits of 80,000 adults (48,000 urban, 32,000 rural) who had died a few years earlier (cases) (Gajalakshmi and Peto, 2004). The other is the baseline survey for an ongoing prospective enquiry of 600,000 adults $(500,000$ urban, 100,000 rural) in the same locations (controls). Follow-up information from the prospective study is not yet available, but those in the baseline survey can be used as controls for the cases in the survey of adult deaths.

Our aim is to assess the relationship, causal or otherwise, of chewing tobacco with mortality at ages 3569 , after excluding from cases and controls who had ever smoked tobacco or drunk alcohol.

\section{Materials and Methods}

The study took place in the late 1990s in the state of Tamil Nadu in South India. There were two study areas, one urban (the city of Chennai, which is the state capital, with a population of 4 million) and one rural (the district of Villupuram, with a population of 2.5 million in about 2000 villages). In both, efforts were made to identify deaths that occurred at the age of 25 and over in urban area during 1995-97 and at the age of $>1$ day in rural area during 1997-98. Made arrangements to visit the home of the dead person(cases for the study) to get information from the family about the educational level, circumstances that led up to the death, smoking, chewing tobacco and 
drinking (alcohol) habits of the dead person before they became ill. The controls were from our surveys of the general population at ages 35 years or above in the same two study areas (from were cases were recruited for the study) during 1998-2001.

\section{Cases for the study}

Urban cases: interviewed 48,000 families: Registration of the fact of death is almost complete in the city of Chennai. Necessary data were abstracted from the death registers in the Vital Statics Department (VSD) in Chennai city to locate the home of the deceased. Since cause of death stated on the death certificate was non-specific in about $50 \%$ of certificates, verbal autopsy(VA) was done, to arrive at underlying cause of death, at the time of visiting home of the deceased to collect required data for the study. The details of the novel verbal autopsy methodology developed and used in Tamil Nadu study was dealt somewhere else (Gajalakshmi et al., 2002; 2003; 2004). This VA methodology consists of training non-medical graduates with at least 15 years of formal education on VA tool to interview the spouse, and/or close associates, and/or neighbours of the deceased and to write the verbal autopsy report. The verbal autopsy report is a narrative description of symptoms and events that led to death and written in local language to enhance the accuracy of the underlying cause of death . All VA reports were reviewed centrally and independently by two medical doctors, unaware of risk factor(s) data, to arrive at the probable underlying cause of death and coded the underlying cause of death according to the 9th International Classification of Diseases (ICD-9) (World Health Organization, 1977).

There were 72,000 deaths among adults aged 25 or older at the time of death during the study period 199597 in Chennai city. Of these, 5000 deaths were attributed to external causes (unintentional injuries, suicide or homicide) and 67,000 deaths to medical causes in the VSD records. Deaths due to external causes were excluded in the study. We were successful in tracing and interviewing 48,000 out of 67,000 households during 1998-1999. 19000 houses could not be visited because the address was missing or inadequate, the house no longer existed, or the family had moved. The cause of death of 1000 deaths that were attributed to medical causes in the VSD records were reclassified to external causes based on the VA report diagnosis, hence excluded from the study. Finally we left with 47,000 cases (27,000 men and 20,000 women) aged 25 or over at the time of death for the urban study. Of this, 5206 men and 8260 women were lifelong non-smoking non-drinkers of age 35-69 at the time of death.

Rural cases: interviewed 32,000 families: The registration of the fact of death is less than $60 \%$ complete in rural Tamil Nadu. Hence efforts were made to identify deaths at all ages (except deaths at age $\leq 1$ day) irrespective of cause of death during 1997-98 in the study area from various sources such as records in the Village Administrative Offices in the study district, enquiring village health nurses /health care workers and village leaders in the study area. Field interviewers were natives of the study area and sought the help of the village leaders and/or village health care workers in obtaining introductions to the relatives, neighbours, or associates of the deceased. Verbal autopsy methodology and, the method of assigning and coding the probable underlying cause of death were as in urban case-control study(Gajalakshmi et al., 2002; 2003; 2004).

The total number of deaths identified during 1997-98 was 40763 . Of these, 1927 deaths could not be traced because the addresses were missing/ incomplete in the Village Administrative Offices or the occupants had moved out after death. Of the 38836 households traced and interviewed, 27,000 deaths (cases: 16,000 men and 11,000 women) were due to medical causes and 5000 were due to external causes at ages 25 or older. Of this, 3278 men and 5716 women were lifelong non-smoking non-drinkers of age 35-69 at the time of death.

Controls for the study are from population surveys conducted in urban (Chennai city) and rural (Villupuram) study areas in Tamil Nadu

Urban population survey: 500,800 individuals: A population survey (Gajalakshmi et al., 2007) was undertaken in Chennai city during 1998-2001. The men and women aged 35 years or over residing in the randomly chosen study area were interviewed at home. Precautions similar to those in the case-control studies were taken to ensure strict quality control of fieldwork, coding, and data entry. Details on the following variables were collected in this population survey: age, sex, educational status, tobacco smoking, tobacco chewing and alcohol drinking. Of 500,816 interviewed, 138,928 men and 219,241 women were lifelong non-smokers and non-drinkers aged 35-69 at the time of baseline survey.

Rural population survey: 100,000: A similar population survey was performed at the same time (1998-2001) in the rural study area. Interviewed all people aged 35 years or over resident in seven of the 22 rural administrative blocks that make up the study area. Of 105,837 interviewed, 23,254 men and 47,883 women were lifelong non-smokers and non-drinkers aged 35-69 at the time of baseline survey.

\section{Quality control}

The survey reports and verbal autopsy reports submitted by the field Interviewers were validated by selecting randomly $5 \%$ of the households for re-interview by the senior investigator. This was done one week after receiving the output from the field Interviewers and blind to its results. The random checking was done partly because knowledge that a revisit might well take place would ensure reliably motivated fieldwork at the initial survey and to identify any systematic defect in the interview techniques. The questionnaires were checked centrally for consistency and missing values by coding clerks, and were double-entered into the computer.

\section{Statistical methods}

In this study chewing was defined as daily chewing of tobacco or related tobacco products, either alone or in combination, for at least 6 months. The term chewer was consistently used to mean ever chewer (former +current). 
This study uses the controls from the population surveys in urban and rural areas since their exposure distribution should have been reasonably representative of the population at risk of becoming cases. In the general population, chewing habits may be associated with smoking and drinking habits. As the risk of chewing is likely to be much smaller than those of smoking and drinking, it is easiest to study them unbiasedly in people who never smoke or drink alcohol. Because the number of cases and of controls, in this study ,is so large, it is possible to restrict attention only to those who never smoked tobacco or drank alcohol.

The urban and rural case-control studies were analysed separately as well as combined. For each category of disease the cases were those who died of it and were compared with population controls; for each different category of disease the control group was always the same. Logistic regression models in STATA (version 8) statistical software (Statacorp LP 2005) were used to calculate mortality odds ratios. The excess death caused by chewing tobacco was calculated by multiplying over all number of deaths among chewers by 1-1/RR, in which $\mathrm{RR}$ is the adjusted mortality odds ratio.

\section{Results}

Detailed analyses of mortality among lifelong nonsmoking non-drinkers are reported here at the age range of 35-69 years because the underlying cause of death assigned by verbal autopsy is more reliable in middle age (35-69) than at older age (70+). A total of 22460 cases (urban: 5206 men and 8260 women; rural: 3278 men and 5716 women) and 429306 controls (urban: 138928 men and 219241 women; rural 23254 men and 47883 women) were analysed.

Table 1 shows key characteristics of 22,460 cases and 429306 controls. The cases were on average 9-11 years older than the controls. A higher proportion of women compared to men did not have formal education in both urban and rural study areas and a higher proportion of participants, both men and women, had no formal education in rural area compared to those in urban area. A higher proportion of cases compared to controls in both sexes and in both study areas were ever chewers of tobacco.

\section{The risk associated with chewing tobacco}

All Cause mortality: Table 2A and 2B give mortality odds ratio adjusted for completed years of education and age comparing ever tobacco chewers with never chewers among lifelong non-smoking non-drinkers in urban and rural areas by sex for various disease categories of medical causes. The risk of dying from all medical causes combined was higher among chewers in rural study area (rural men:RR-1.3 [95\%CI:1.2-1.5] and rural women:RR-1.6 [95\%CI:1.5-1.7]) than in urban study area (urban men:RR-1.2 [95\%CI:1.1-1.3] and urban women:RR-1.3 [95\%CI:1.2-1.4]) in both sexes. Table 3 shows when men and women from urban and rural study areas are combined , the ever-chewer mortality odds ratio for death from all medical causes combined, additionally adjusted for sex and study area, was $1.3(95 \%$ CI: 1.2-1.4).

Table 1. Characteristics of 22460 Cases and 429306 Controls that Never Smoked Tobacco and Never Drank Alcohol

\begin{tabular}{|c|c|c|c|c|c|c|c|c|}
\hline & \multicolumn{4}{|c|}{ Urban } & \multicolumn{4}{|c|}{ Rural } \\
\hline & \multicolumn{2}{|c|}{ Men } & \multicolumn{2}{|c|}{ Women } & \multicolumn{2}{|c|}{ Men } & \multicolumn{2}{|c|}{ Women } \\
\hline & Cases & Controls & Cases & Controls & Cases & Controls & Cases & Controls \\
\hline Number & 5206 & 138928 & 8260 & 219241 & 3278 & 23254 & 5716 & 47883 \\
\hline Age in yrs: Mean(SD) & $57(9)$ & $46(9)$ & $57(9)$ & $47(9)$ & $56(9)$ & 47(10) & $56(9)$ & 46(9) \\
\hline No formal education $\%$ & 17.4 & 6.1 & 47.6 & 29.2 & 78.1 & 46.5 & 89.3 & 84.1 \\
\hline Ever chewed tobacco $\%$ & 7.9 & 4.8 & 19.5 & 10.1 & 44.7 & 21.2 & 60.2 & 36.1 \\
\hline \multicolumn{9}{|c|}{$\begin{array}{l}\text { Table 2A. Mortality Odds Ratios of Diseases Associated with Chewing Tobacco at Ages 35-69 Yrs by Urban/ } \\
\text { Rural Location in Non-smoking Non-drinking Men in Tamil Nadu, South India }\end{array}$} \\
\hline \multirow[t]{2}{*}{ Cause of death(ICD 9) } & \multicolumn{2}{|c|}{$\begin{array}{l}\text { Cases Never/ever } \\
\text { tobacco chewers }\end{array}$} & \multicolumn{2}{|c|}{$\mathrm{RR}^{*}(95 \% \mathrm{CI})$} & \multicolumn{2}{|c|}{$\begin{array}{l}\text { Cases Never/ever } \\
\text { tobacco chewers }\end{array}$} & \multicolumn{2}{|c|}{$\mathrm{RR}^{*}(95 \% \mathrm{CI})$} \\
\hline & \multicolumn{4}{|c|}{ Urban Men } & \multicolumn{4}{|c|}{ Rural Men } \\
\hline Respiratory & \multicolumn{2}{|c|}{$428 / 41$} & \multicolumn{2}{|c|}{$1.0(0.7-1.4)$} & \multicolumn{2}{|c|}{$223 / 186$} & \multicolumn{2}{|c|}{$1.3(1.1-1.6)$} \\
\hline Tuberculosis & \multicolumn{2}{|c|}{$225 / 19$} & \multicolumn{2}{|c|}{$0.9(0.6-1.5)$} & \multicolumn{2}{|c|}{$145 / 129$} & \multicolumn{2}{|c|}{$1.5(1.2-2.0)$} \\
\hline Other respiratory & \multicolumn{2}{|c|}{$203 / 22$} & \multicolumn{2}{|c|}{$1.1(0.7-1.8)$} & \multicolumn{2}{|c|}{$78 / 57$} & \multicolumn{2}{|c|}{$1.0(0.7-1.5)$} \\
\hline Cancer & \multicolumn{2}{|c|}{$514 / 55$} & \multicolumn{2}{|c|}{$1.4(1.0-1.8)$} & \multicolumn{2}{|c|}{$64 / 50$} & \multicolumn{2}{|c|}{$1.5(1.0-2.3)$} \\
\hline Upper aerodigestive & \multicolumn{2}{|c|}{$113 / 21$} & \multicolumn{2}{|c|}{$2.2(1.4-3.6)$} & \multicolumn{2}{|c|}{$14 / 15$} & 1.9( & $0.9-4.3)$ \\
\hline Stomach & $69 / 1$ & & $1.9(0.9$ & $3.6)$ & $18 /$ & & 2.1( & $1.1-4.2)$ \\
\hline Other cancer site & $332 / 2$ & & $0.9(0.6$ & $1.4)$ & 32 & & 0.9( & .6-1.9) \\
\hline Vascular & $2614 / 20$ & & $1.0(0.8$ & 1.2) & 587 & & 1.2( & $.0-1.4)$ \\
\hline Stroke & $260 / 24$ & & $1.1(0.7$ & 1.7) & $76 /$ & & 2.2( & (.6-3.0) \\
\hline Cardiac \& Other vascular & $2354 / 18$ & & $1.0(0.9$ & $1.2)$ & $511 /$ & & 1.0( & $0.9-1.2)$ \\
\hline Other medical & $1238 / 11$ & & $1.0(0.9$ & $1.3)$ & 939 & & 1.4( & $1.2-1.5)$ \\
\hline Unspecified medical & $305 / 32$ & & $1.0(0.7$ & 1.4) & 359 & & 1.3( & 1.1-1.6) \\
\hline Other specified medical & $933 / 78$ & & $1.1(0.9$ & $1.4)$ & 580 & & 1.4( & $.2-1.6)$ \\
\hline All medical causes & $4794 / 41$ & & $1.2(1.1$ & 1.3) & $1813 /$ & 465 & 1.3( & $.2-1.5)$ \\
\hline
\end{tabular}

*Adjusted for age and educational level 
Table 2B. Mortality Odds Ratios of Diseases Associated with Chewing Tobacco at Ages 35-69 Yrs by Urban/ Rural Location in Non-smoking Non-drinking Women in Tamil Nadu, South India

\begin{tabular}{|c|c|c|c|c|}
\hline \multirow[t]{2}{*}{ Cause of death(ICD 9) } & \multicolumn{2}{|l|}{$\begin{array}{l}\text { Cases Never/ever } \\
\text { tobacco chewers }\end{array}$} & \multicolumn{2}{|l|}{$\begin{array}{l}\text { Cases Never/ever } \\
\text { tobacco chewers }\end{array}$} \\
\hline & \multicolumn{2}{|c|}{ Urban Women } & \multicolumn{2}{|c|}{ Rural Women } \\
\hline Respiratory & $619 / 185$ & $1.4(1.2-1.7)$ & $308 / 538$ & $1.8(1.6-2.1)$ \\
\hline Tuberculosis & $284 / 90$ & $1.6(1.2-2.0)$ & $171 / 295$ & $2.1(1.7-2.6)$ \\
\hline Other respiratory & $335 / 95$ & $1.3(1.1-1.7)$ & $137 / 243$ & $1.6(1.3-1.9)$ \\
\hline Cancer & $1075 / 289$ & $1.6(1.4-1.8)$ & $238 / 295$ & $1.7(1.4-2.1)$ \\
\hline Upper aerodigestive & $134 / 69$ & $2.7(2.0-3.7)$ & $24 / 66$ & 3.8(2.3-6.4_) \\
\hline Stomach & $102 / 34$ & $1.8(1.2-2.7)$ & $46 / 47$ & $1.4(0.9-2.2)$ \\
\hline Breast & $244 / 18$ & $0.5(0.3-0.8)$ & $30 / 23$ & $0.9(0.5-1.7)$ \\
\hline Cervix & $211 / 78$ & $2.0(1.5-2.7)$ & $48 / 84$ & $2.2(1.5-3.2)$ \\
\hline Other cancer sites & $384 / 90$ & $1.5(1.2-1.9)$ & $90 / 75$ & $1.3(1.0-1.9)$ \\
\hline Vascular & $2979 / 599$ & $1.0 .0 .9-1.1)$ & $553 / 677$ & $1.2(1.1-1.4)$ \\
\hline Stroke & $328 / 91$ & $1.3(1.1-1.7)$ & $151 / 239$ & $1.3(1.0-1.6)$ \\
\hline Cardiac \& Other vascular & $2651 / 508$ & $1.0(0.9-1.1)$ & $402 / 438$ & $1.2(1.1-1.4)$ \\
\hline Other medical & $1975 / 539$ & $1.3(1.2-1.4)$ & $1175 / 1932$ & 1. $6(1.5-1.8)$ \\
\hline Unspecified medical & $712 / 259$ & $1.5(1.3-1.7)$ & $377 / 744$ & $1.9(1.7-2.2)$ \\
\hline Other specified medical & $1263 / 280$ & $1.2(1.0-1.4)$ & $798 / 1188$ & $1.5(1.4-1.7)$ \\
\hline All medical causes & $6648 / 1612$ & $1.3(1.2-1.4)$ & $2274 / 3442$ & $1.6(1.5-1.7)$ \\
\hline
\end{tabular}

*Adjusted for age and educational level

Table 3. Mortality Odds Ratios of Diseases Associated with Chewing Tobacco at Ages 35-69 Yrs Combined Men and Women, Non-smoking and Non-drinking Data of Urban and Rural Study Areas in Tamil Nadu, South India

\begin{tabular}{lcc}
\hline Cause of death(ICD 9) & $\begin{array}{c}\text { Cases } \\
\text { Never/ever } \\
\text { tobacco chewers }\end{array}$ & RR*(95\% CI) \\
\hline Respiratory & $1578 / 950$ & $1.5(1.4-1.7)$ \\
Tuberculosis & $825 / 533$ & $1.7(1.5-1.9)$ \\
Other respiratory & $753 / 417$ & $1.4(1.2-1.6)$ \\
Cancer & $1891 / 689$ & $1.5(1.4-1.7)$ \\
Vascular & $6733 / 1898$ & $1.1(1.0-1.2)$ \\
Stroke & $815 / 475$ & $1.4(1.2-1.6)$ \\
Cardiac \& Other vascular & $5918 / 1423$ & $1.0(0.9-1.1)$ \\
Other medical & $5327 / 3394$ & $1.4(1.3-1.5)$ \\
Unspecified medical & $1753 / 1357$ & $1.5(1.3-1.6)$ \\
Other specified medical & $3574 / 2037$ & $1.4(1.3-1.5)$ \\
All medical causes & $15529 / 6931$ & $1.3(1.2-1.4)$ \\
\hline
\end{tabular}

*Adjusted for age,sex ,educational level and study area (unban/ rural)

Mortality of selected diseases

Table 2A shows among urban men the age and education adjusted mortality odds ratio was significant (weakly) (RR-1.4, 95\%CI: 1.0-1.8) only for all cancer sites combined whereas in rural men it was significant for respiratory diseases combined (RR-1.3,95\%CI:1.1-1.6), respiratory tuberculosis (RR-1.5, 95\% CI: 1.2-2.0) and stroke(RR-2.2,95\%CI:1.6-3.0), and weakly significant for all cancer sites combined (RR-1.5,95\%CI:1.0-2.3). Of the cancers, age and education adjusted mortality odds ratios were significantly higher for upper aerodigestive cancers(RR-2.2, 95\%CI:1.4-3.6) in urban men and for stomach cancer (RR-2.1, 95\%CI: 1.1-4.2) in rural men.

Table 2B shows among both urban and rural women the age and education adjusted mortality odds ratios were statistically significant for respiratory diseases combined (urban: RR-1.4, 95\%CI: 1.2-1.7, Rural: RR-1.8, 95\%CI: 1.6-2.1), respiratory tuberculosis (urban:RR-1.6, 95\%CI:
1.2-2.0, Rural: (RR-2.1, 95\%CI: 1.7-2.6), all cancer sites combined (urban:RR-1.6,95\%CI: 1.4-1.8, Rural: RR-1.7, 95\%CI: 1.4-2.1) and stroke (urban:RR-1.3, 95\%CI: 1.11.7, Rural: [weakly significant]RR-1.3,95\%CI: 1.0-1.6). In addition to this, age and education adjusted mortality odds ratio was statistically significant for vascular diseases combined without stroke for rural women (RR-1.2, 95\% CI: 1.1-1.4). Of the cancers, the age and education adjusted mortality odds ratios were significantly higher for stomach cancer only in urban women (RR-1.8,95\%CI: 1.2-2.7) and for the following cancers in both urban and rural women: upper aerodigestive (urban: RR-2.7, 95\%CI: 2.0-3.7; rural: RR-3.8, 95\%CI: 2.3-6.4), cervical cancer (urban: RR-2.0, 95\%CI: 1.5-2.7; rural: RR-2.2, 95\%CI: 1.5-3.2),

Table 3 shows the age, sex, education and study area(urban/rural) adjusted mortality odds ratio in ever tobacco chewers compared to never chewers was significant for deaths from respiratory diseases combined (RR:1.5, 95\% CI:1.4-1.7), respiratory tuberculosis (RR:1.7, 95\%CI:1.5-1.9), cancers(all sites combined) (RR:1 .5,95\%CI:1.4-1.7) and stroke(RR:1.4, 95\%CI:1.21.6).

\section{Discussion}

Tobacco chewing is not considered as stigma in India. Hence we do not expect any misclassification of tobacco chewing habit in this study, even though the data were collected on dead people from the surviving family members. Since the analyses excluded smokers and drinkers the associations seen in this study could not be confounded by tobacco smoking or alcohol drinking, but they could, despite adjustment for educational level, be residually confounded by social factors.

Of the 22,460 deaths from all medical causes at ages $35-69,31 \%$ were ever chewers and $7.1 \%$ of deaths would have been avoided if ever chewers had non-chewers death rates. Significantly higher mortality odds ratio, adjusted for 
age, sex, education and study area, with tight confidence interval, was found for all medical causes combined among ever chewers of tobacco ( RR: 1.3 (1.2-1.4); the corresponding mortality odds ratio when men and women were combined in urban study area was $1.2(95 \% \mathrm{CI}: 1.1-$ 1.3) and in rural study area 1.5(95\% CI:1.4-1.6) compared to never chewers. Similar magnitude of risk of death for all medical causes associated with smokeless tobacco use was observed in a cohort of construction workers aged 35 and over in Sweden (RR:1.4, 95\% CI: 1.3-1.8) (Bolinder et al.,1994), in 2 large prospective cohort studies with individuals aged 35 and over in USA(CPS-I:hazard ratio [HR]:1.17, 95\% CI:1.11-1.23 and CPS-II:HR: 1.18, 95\% CI: 1.08-1.29) (Henley et al., 2005), among men 35 and over in the cohort study in Mumbai, India ( RR:1.5, 95\% CI: 1.12-2.03) (Gupta et al., 2005) and in the metaanalysis conducted in India, adjusted for smoking, age and education (Females- RR:1.3(1.27-1.42) and MalesRR:1.2(1.05-1.42)) (Sinha et al., 2014).

The present study shows that ever-chewer mortality odds ratio adjusted for age and education for tuberculosis was 1.5-fold in rural men, 2-fold in rural and in urban women compared to never chewers. When both study areas were combined the age, sex, education and study area adjusted ever-chewer mortality odds ratio for tuberculosis was $1.7(1.5-1.9)$ and for respiratory diseases other than tuberculosis was 1.4(1.2-1.6). The present study results are consistent with the Cancer Prevention Study I in USA (Henley et al., 2005) that observed elevated hazard ratios among smokeless tobacco users for respiratory diseases combined (HR:1.28,95\% CI:1.03-1.59) and the Mumbai cohort study (Gupta et al., 2005) in India that noted about $40-46 \%$ higher risk for death from tuberculosis among tobacco chewers in both genders. The reason for this increased risk among tobacco chewers for respiratory diseases, including tuberculosis, is not clear.

In the urban study, cause of death due to cancer based on VA reports was confirmed with medical records (Gajalakshmi et al., 2004) and this was not feasible in rural study area. Among tobacco chewers the risk of death from cancer was about $40-50 \%$ higher in men and 60-70\% higher in women compared to never chewers. The age and education adjusted mortality odds ratio associated with tobacco chewing was significant for deaths from upper aerodigestive, stomach and cervical cancers. Of the cancers mentioned above, except cervical cancer (McCann et al., 1992;Gajalakshmi et al., 2012), cancers in other sites are well known to be caused by chewing tobacco (Stockwell and Lyman, 1986; Sankaranarayanan et al., 1989; Gupta et al., 1980; Rao et al., 1994; Dikshit and Kanhere, 2000; Balaram et al., 2002; Znaor et al., 2003; ; IARC Monographs 2004, 2007; Gupta et al., 2005; Henley et al., 2005; Phukan et al., 2005; Razmara et al., 2013).

Noted increased heart rate and high blood pressure in tobacco chewers (Stockwell and Lyman, 1986; Bolinder and de Faire, 1998) and high levels of total cholesterol, low-density lipoprotein cholesterol and triglycerides in tobacco smokers and in tobacco chewers compared to never users of tobacco (Nanda and Sharma, 1988). A study conducted in Sweden (Bolinder et al., 1994) among men noted the relative risk of 1.4(1.2-1.6) for cardiovascular diseases in smokeless tobacco users. Mumbai cohort study (Gupta et al., 2005) in India found elevated risk of death from vascular diseases among women tobacco chewers only. Both in CPS I and II elevated risk of death from cardiovascular disease was found among smokeless tobacco users (CPS-I: Hazard ratio(HR):1.18, 95\%CI:1.11-1.26 and CPS-II:HR:1.23,95\%CI:1.09-1.39) (Henley et al., 2005). The present study shows higher age and education adjusted mortality risk, of death from stroke among tobacco chewers compared to never chewers in rural men 2.2(1.6-3.0), in rural women 1.3(1.0-1.6) and in urban women 1.3(1.1-1.7).However, the mortality odds ratio (men, women and study areas combined) adjusted for age, sex, education and study area for stroke was 1.4(1.21.6) and for vascular diseases 1.1(1.0-1.2).

The strengths of the study are large sample size, inclusion of all deaths that occurred in the study areas, having used novel verbal autopsy method (with strict supervision and quality control) to assign the cause of death for all cases(deaths), use of population controls from general population surveys conducted in the areas from where cases were recruited for the study, and exclusion of smokers and alcohol drinkers from the analyses to avoid confounding by tobacco smoking and drinking alcohol.

In conclusion, the present study is the first large study in India on lifelong non-smoking non-drinkers at ages 35-69. The age, sex, education and study area adjusted mortality odds ratio was about $30 \%$ higher in ever-chewers in south India and the risk is higher among those in rural area compared to those in urban area. Chewing tobacco is the cause of $7.1 \%(n=1595)$ of deaths from all medical causes among non-smoking non-drinkers at ages 35-69 in south India. Of the cancers, ever-chewer mortality odds ratio adjusted for age and education was significant for upper aerodigestive cancers in urban men, urban women and in rural women, stomach cancer in rural men and urban women and, cervical cancer in both rural and urban women. The reason for increased risk among ever tobacco chewers for stroke and respiratory diseases including tuberculosis found in this study are not clear.

\section{Acknowledgements}

Funded by direct support from the UK Medical Research Council and Cancer Research UK to the University of Oxford Clinical Trial Service Unit and Epidemiological Studies Unit, University of Oxford with additional support from the Fogarty International Center, Bethesda, MD, USA. The field work was started when VG was at the Cancer Institute (WIA) in Chennai. We thank the Chennai and Villupuram district Vital statistics division for providing mortality data and all study participants.

\section{References}

Balaram P, Sridhar H, Rajkumar T, et al (2002). Oral Cancer in Southern India: the influence of smoking, drinking, paan chewing and oral hygiene. Int J Cancer, 98, 440-5.

Bolinder G, Alfredsson I, England A, de Faire U (1994). Smokeless tobacco use and increased cardiovascular mortality among Swedish construction workers. Am J Public 
Health, 84, 399-404.

Bolinder G, de Faire U (1998). Ambulatory 24-h blood pressure monitoring in healthy, middle-aged smokeless tobacco users, smokers, and nontobacco users. Am J Hypertens, 11, 1153-63.

Dikshit RP, Kanhere S (2000). Tobacco habits and risk of lung, oropharyngeal and oral cavity cancer: a population-based case-control study in Bhopal, India. Int J Epidemiol, 29, 609-14.

Gajalakshmi V, Peto R (2004).Verbal autopsy of 80,000 adult deaths in Tamilnadu, South India. BMC Public Health, $4,47$.

Gajalakshmi V, Peto R, Kanimozhi CV, Whitlock G, Veeramani $\mathrm{D}$ (2007). The Chennai prospective study of mortality among 500,000 adults in Tamil Nadu, South India. Int J Epid, 36, 1190-95.

Gajalakshmi V, Peto R, Kanaka S, Balasubramanian S (2002). Verbal autopsy of 48,000 adult deaths attributable to medical causes in Chennai (formerly Madras), India. BMC Public Health, 2, 7.

Gajalakshmi V, Peto R, Kanaka S, Jha P (2003). Smoking and mortality from tuberculosis and other diseases in India: retrospective study of 43,000 adut male deaths and 35,000 controls. Lancet, 362, 507-15

Gajalakshmi V, Whitlock G, Peto R (2012). Social inequalities, tobacco chewing, and cancer mortality in south India:a casecontrol analysis of 2580 cancer deaths among non-smoking non-drinkers. Cancer Causes Control, 23, 91-8

Global progress report on implementation of the WHO FCTC (2010). Geneva, World Health Organization, 2010.Available from: http://www.who.int/entity/fctc/reporting/progress_ report_final.pdf.

Gupta PC, Mehta FS, Daftary DK et al (1980). Incidence rates of oral cancer and natural history of oral precancerous lesions in a 10-year follow-up study of Indian villages. Community Dent Oral Epidemiol, 8, 6-333

Gupta PC, Pednekar MS, Parkin DM, Sankaranarayanan R (2005). Tobacco associated mortality in Mumbai (Bombay) India. Results of the Bombay cohort study. Int J Epidemiol; 34, 1395-402

Henley SJ, Thun MJ, Connell C, Calle EE (2005). Two large prospective studies of mortality among men who use snuff or chewing tobacco (United States). Cancer Causes Control, 16, 347-58

IARC monographs on the evaluation of carcinogenic risks to humans. smokeless tobacco and some tobacco-specific N-Nitrosamines. Volume 87, Lyon, France, 2007.

IARC monographs on the evaluation of carcinogenic risks to humans. Tobacco smoke and involuntary smoking. Volume 83. Lyon, France: IARC, 2004

McCann MF, Irwin DE, Walton LA, et al (1992). Nicotine and cotinine in the cervical mucus of smokers, passive smokers, and nonsmokers. Cancer Epidemiol Biomarkers Prev, 1, $125-9$

Nanda PK, Sharma MM (1988). Immediate effect of tobacco chewing in the form of 'paan' on certain cardio-respiratory parameters. Indian J Physiol Pharmacol, 32, 105-13.

Phukan RK, Zomawia E, Narain K, Hazarika NC, Mahanta J (2005). Tobacco use and stomach cancer in Mizoram, India. Cancer Epidemiol Biomarkers Prev, 14, 1892-6

Rao DN, Ganesh B, Rao RS et al (1994). Risk assessment of tobacco, alcohol and diet in oral cancer: a case-control study. Int J Cancer, 58, 469-73.

Razmara A , Madani AH, Daneshnia F (2013). Effect of smokeless tobacco on oral cancer: A case-control study. Life Sci J, 10, 155-9

Sankaranarayanan R, Duffy SW, Padmakumary G, et al (1989). Tobacco chewing, alcohol and nasal snuff in cancer of the gingival in Kerala, India. Br J Cancer, 60, 638-43

Sinha DN, Palipudi KM, Gupta PC, et al (2014). Smokeless tobacco use: A meta-analysis of risk and attributable mortality estimates for India. Indian J Cancer, 51, 73-7.

Statacorp LP (2005). Stata statistical software: release 8.0. statacorp lp, college station, TX 77845, USA

Stockwell HG, Lyman GH (1986). Impact of smoking and smokeless tobacco on the risk of cancer of the head and neck. Head Neck Surg, 9, 104-10

Thakur JS, Prinja S, Bhatnagar N, Rana S, Sinha DN (2013). Socioeconomic inequality in the prevalence of smoking and smokeless tobacco use in India. Asian Pac J Cancer Prev, 14, 6965-9.

World Health Organization (1977). Manual of International Classification of Diseases, Injuries and Causes of Death, 9th Rev, Geneva.

World Health Organization (1999). Making a Difference. In World Health Report. Geneva.

Znaor A, Brennan P, Gajalakshmi V, et al (2003). Independent and combined effects of tobacco smoking, chewing and alcohol drinking on the risk of oral, pharyngeal and esophageal cancers in Indian men. Int J Cancer, 105, 681-86. 